\title{
Contribution of Ruptured Uterus to Maternal Mortality in Rural South Eastern Nigeria.
}

\author{
Ugochukwu O.J. Umeora, Brown N Ejikeme and Vincent E Egwuatu \\ Department of Obstetrics \& Gynaecology, Ebonyi State University Teaching Hospital. \\ Abakaliki - Ebonyi State, Nigeria.
}

\begin{abstract}
Context: Maternal mortality statistics in Nigeria derive mainly from urban based hospital data. In rural areas of the country where available medical facilities and trained medical personnel are inadequate, the incidence, and major causes of maternal death may differ.

Aims and Objectives: To provide information on maternal death in an entirely rural setting in Southeast Nigeria, and determine contribution of uterine rupture to maternal mortality.

Methods: Analysis of records of maternal deaths and ruptured uterus over a 10-year period in a rural Nigerian community.

Main Outcome Measure: Maternal deaths resulting from ruptured uterus

Results: The Maternal Mortality ratio was $2659 / 100000$ maternities - 789/100,000 for booked and $8235 / 100,000$ for unbooked women. Ruptured uterus accounted for $31.9 \%$ of deaths. Haemorrhage, obstructed labour, sepsis and eclampsia accounted for $29.2,13.9,12.5$ and $8.3 \%$ of the deaths respectively. A patient each died from complications of abortion and anaesthesia. Perinatal mortality rate was $963 / 1000$ births.

Conclusion: Ruptured uterus and obstructed labour were responsible for over half of the maternal deaths. High MMR and PNMR reflect paucity of maternal and public health services in the rural areas.
\end{abstract}

Key Words: Maternal mortality, ruptured uterus, rural setting, Southeast Nigeria.

\section{Introduction}

Maternal mortality has continued to rise in sub-Saharan Africa in spite of the launching of the global Safe Motherhood Initiative in Nairobi, Kenya in $1987^{\prime}$. The maternal mortality ratio of 1000 per 100,000 deliveries often quoted for Nigeria ${ }^{2}$ is derived mainly from figures from urban based hospital studies. In the rural areas MMR appear much higher ${ }^{3,4 .}$ Obstetric haemorrhage remains the major cause of maternal mortality globally ${ }^{5}$. In the developed countries, uterine rupture is rare and has an incidence of about 1 in 1,500 deliveries ${ }^{6}$. In the developing world, on the contrary, obstructed labour and uterine rupture are common and together impact significantly on the $\mathrm{MMR}^{7.9}$. The present review evaluates the relative contribution of ruptured uterus to maternal mortality in a purely rural Nigerian community.

\section{Materials and Methods}

Over the 10 year study interval, January 1994 to December 2003, St. Vincent's hospital, Ndubia, in Ebonyi State of Eastern Nigeria was staffed with a qualified resident Obstetrician and Gynaecologist, medical Officers, nursing staff and other personnel, and was a training centre for Community Health workers. It was equipped with functional ultrasound and laboratory facilities, and served as a referral centre and first point of contact, with orthodox medicine for the surrounding poor, illiterate and mainly agrarian communities of that region of Ebonyi State and the neighbouring Cross River State. Blood transfusion services were unreliable and communication facilities, non existent. Road transportation was limited, and the roads were generally unmotorable during the rainy season. Labour usually started at home, and was mostly supervised by an illiterate Traditional Birth Attendant (TBA) or Spiritualist. Unsuccessful labour was usually accompanied by much delay before the arrival of the women into hospital.

Three thousand and forty-six women were delivered in the hospital during the study interval, and includec 93 cases of ruptured uterus and 81 maternal deaths. The socio demographic characteristics of the women who suffered uterine rupture and/or died were obtained from the case records. Details and outcome of labour, causes of death and possible risk factors for uterine rupture were examined and the results analyzed using the epi info statistical software package version 3.2.

\section{Results}

Eighty-one maternal deaths occurred of a total of 3046 deliveries giving a total Maternal Mortality Ratio (MMR) of 2,659 per 100,000 maternities. The records of 72 maternal deaths were complete and were analyzed. The difference between the MMR for the booked women $(789 / 100,000)$ and the unbooked $(8235 / 100,000)$ was statistically significant.

Corrrespondence: Dr. U. O. J. Umeora, P.O. Box 980, Abakaliki 480001, Ebonyi State. Nigeria.

Email:oujair@yahoo.com 
$\left(X^{2}=122.67\right.$ and $p$ value $\left.<0.05\right)$. The causes of maternal death are shown on Table 1. Ruptured uterus was the leading cause accounting for $23(31.9 \%)$ of the deaths. Haemorrhage, obstructed labour, sepsis and eclampsia/pre eclampsia were responsible for 29.2 , $13.9,12.5$ and $8.3 \%$ of maternal deaths respectively. A patient each died from abortion related and anaesthetic complications. The cause of death was not stated in one case.

\section{Table 1}

Causes of Maternal Mortality 1994 - 2003. $(n=72)$

\begin{tabular}{lrr}
\hline \multicolumn{1}{c}{ Causes } & n & $\%$ \\
\hline Haemorrhage & 21 & 29.17 \\
Ruptured Uterus & 23 & 31.94 \\
Sepsis & 9 & 12.50 \\
Obstructed Labour & 10 & 13.89 \\
Pre eclampsia/Eclampsia & 6 & 8.33 \\
Abortion complications & 1 & 1.39 \\
Anaesthetic complications & 1 & 1.39 \\
Not stated & 1 & 1.39 \\
TOTAL & 72 & 100.00 \\
\hline
\end{tabular}

Ninety-three patients with ruptured uteri were admitted during the study period. The records of 82 of them were complete and were analyzed. The socio demographic characteristics of the women are displayed on Table 2 . Over $84 \%$ of the women were unbooked and this group accounted for 21 of the 23 deaths. The remaining 2 deaths occurred among the $13(15.85 \%)$ booked cases. The majority of the women with ruptured uteri $(47.6 \%)$ were aged between 25 and 29 years. No teenage mother suffered uterine rupture. Nearly $82 \%$ of the patients were of parity $1-4$. The 2 primigravid women with ruptured uterus survived. Nine women lived within 5 $\mathrm{km}$ of the hospital, 20 within $10 \mathrm{~km}$ and 32 farther than $10 \mathrm{~km}$. Corresponding numbers of maternal deaths for the groups were 3,3 , and 8 respectively. Nine deaths occurred among the 21 women whose home hospital distance was undetermined. All the patients with ruptured uterus belonged to social classes IV and V.

The risk factors for uterine rupture are indicated on Table 3. A previous uterine scar was the commonest risk factor and was found in $32(39.02 \%)$ of the women. Other risk factors included use of oxytocics in 21 (25.6\%) patients, obstructed labour in $10(12.2 \%)$ and malpresentation in $8(9.76 \%)$ women. No obvious risk factors were identified in 11 patients, 8 of whom were grandmultiparous (para $\geq 5$ ). Spontaneous uterine rupture occurred in $56(68.3 \%)$ women, and the rupture was traumatic in $26(31.7 \%)$ patients. Table IV presents the Diagnosis Surgery interval and the surgical treatment of the patients. Eleven patients $(13.4 \%)$ died without any surgical intervention. In 9, 18, and 44 women, operation was carried out within 6 hours, 713 hours, and 14 hours and over respectively. No death occurred among women who were operated upon within 6 hours of uterine rupture. Of the 44 patients who had surgery over 14 hours after uterine rupture, 10 $(22.7 \%)$ died. This accounted for $43.5 \%$ of the mortality.

The majority of the women (33 out of 82) underwent subtotal hysterectomy and 9 died. (Table 4). Total abdominal hysterectomy was performed in 6 patients, one of whom died. Of the 19 women who had repair of the ruptured uterus and bilateral tubal ligation, 2 died. None of the 13 patients who had uterine repair alone died. None of the 11 women who received no surgical treatment survived. Eighteen patients (21.95\%) received no blood transfusion and included the 11 who died before surgical operation could be performed. Thirty-two (39\%) of the women were transfused with 1 2 pints of blood, $21(25.6 \%)$ with $3-4$ units, and 11 $(13.4 \%)$ with 5 or more units. Only 4 babies were delivered alive, and one died in the early neonatal period. The perinatal mortality rate was 963 per 1000 births.

\section{Discussion}

A horrifyingly high MMR of 2659/100,000 maternities found in this study is more than double the very high Nigerian national average of $1000 / 100,000^{5}$. Nigeria has one of the highest MMR of any developing country in the world, a figure about 100 times that of the United Kingdom. Uterine rupture and obstructed labour together accounted for over half of the maternal deaths in this review (Table I), reflecting, as observed in other reports ${ }^{10}$ the poor quality Obstetric care available in the rural areas.

Uterine rupture occurred in 93 of the 3046 deliveries, an incidence of $1: 33$, and was the leading cause of maternal death in this study (Table I) accounting for $31.9 \%$ of cases. In this study, $21(91.3 \%)$ of the 23 women that died following uterine rupture were unbooked and had no antenatal care elsewhere. Obstetric haemorrhage, the largest contributor to maternal death globally, was the second leading cause $(29.2 \%)$ in our series. In the rural communities, labour usually starts at home, supervised by an illiterate, untrained Traditional Birth Attendant (TBA). Difficult or unsuccessful labour is invariably associated with considerable delay in arrival of the women to the hospital. Most cases of obstructed labour occur in rural areas, the uterus rupturing before the patients arrive in the hospital ${ }^{11}$. Where the uterus is still intact, patients usually come into the hospital in late second stage of labour, exhausted, infected, with the fetus already dead, the uterus in tonic contraction and rupture imminent ${ }^{12}$. In such circumstances, immediate delivery becomes mandatory, and in rural areas, 
Table 2

Socio Demographic Characteristics of 82 Women with Ruptured Uteri.

\begin{tabular}{|c|c|c|c|c|c|}
\hline \multirow{2}{*}{ Parameters } & & \multirow[b]{2}{*}{$\mathbf{n}$} & \multirow[b]{2}{*}{$\%$} & \multicolumn{2}{|c|}{ MORTALITY } \\
\hline & & & & $\mathbf{n}$ & $\%$ \\
\hline \multirow[t]{5}{*}{ Age(years) } & $\leq 19$ & - & - & - & - \\
\hline & 2024 & 18 & 21.95 & 8 & 34.8 \\
\hline & 2529 & 39 & 47.56 & 9 & 39.1 \\
\hline & 3034 & 19 & 23.17 & 4 & 17.4 \\
\hline & $\geq 35$ & 6 & 7.32 & 2 & 8.7 \\
\hline \multirow[t]{2}{*}{ Booking Status } & Booked & 13 & 15.85 & 2 & 8.7 \\
\hline & Unbooked & 69 & 84.15 & 21 & 91.3 \\
\hline \multirow[t]{3}{*}{ Parity } & 0 & 2 & 2.44 & 0 & 0 \\
\hline & 14 & 67 & 81.71 & 17 & 74.0 \\
\hline & $\geq 5$ & 13 & 15.85 & 6 & 26.0 \\
\hline Residence & $\leq 5$ & 9 & 10.98 & 3 & 13.04 \\
\hline \multirow[t]{3}{*}{$(\mathrm{km})$} & $\overline{6} 10$ & 20 & 24.39 & 3 & 13.04 \\
\hline & $\geq 10$ & 32 & 39.02 & 8 & 34.78 \\
\hline & determined & 21 & 25.61 & 9 & 39.11 \\
\hline \multirow[t]{5}{*}{ Social Class } & I & - & $=$ & - & - \\
\hline & II & - & - & - & - \\
\hline & III & - & - & - & - \\
\hline & IV & 5 & 6.1 & 2 & 8.7 \\
\hline & V & 77 & 93.9 & 21 & 91.3 \\
\hline
\end{tabular}

Table 3

Risk Factors for Uterine Rupture $(\mathrm{N}=\mathbf{8 2})$

\begin{tabular}{lrr}
\hline Factors & $\mathbf{n}$ & \multicolumn{1}{c}{$\%$} \\
\hline Previous scar & 32 & 39.02 \\
Oxytocics & 21 & 25.61 \\
Malpresentation & 8 & 9.76 \\
Obstructed labour & 10 & 12.20 \\
None identified & 11 & 13.41 \\
Total & 82 & 100.00 \\
\hline
\end{tabular}

Table 4

Surgical Treatment of 82 Women with Ruptured Uterus.

\begin{tabular}{lrrrr}
\hline Intervention & \multicolumn{4}{c}{ Mortality } \\
& n & $\%$ & n & $\%$ \\
\hline Surgery Performed & & & & \\
$\quad$ Subtotal Hysterectomy & 33 & 40.24 & 9 & 39.1 \\
$\quad$ Total Hysterectomy & 6 & 7.32 & 1 & 4.4 \\
Repair with BTL & 19 & 23.17 & 2 & 8.7 \\
Repair alone & 13 & 15.85 & 0 & 0 \\
No surgery & 11 & 13.41 & 11 & 47.8 \\
& & & & \\
Intervention Time (hours) & & & & \\
$\quad$ None & 11 & 13.41 & 11 & 47.8 \\
$\quad 0-6$ & 9 & 10.98 & 0 & 0 \\
$7-13$ & 18 & 21.95 & 2 & 8.7 \\
$\geq 14$ & 44 & 53.66 & 10 & 43.5 \\
\hline
\end{tabular}

classical Caesarean section is sometimes resorted to". It was not surprising therefore, that of the 32 ruptured Caesarean Section scars, 9 were of the upper segment, and that only 4 babies were delivered alive, one of whom died in the early neonatal period.

The surgical treatment of choice in this study, as in other reports ${ }^{8,9,13.14}$ was sub total hysterectomy. The choice of surgical procedure to be performed in each case was determined by clinical expediency, the surgeon's experience availability of a competent Anaesthetist and blood for transfusion. Other contributory deciding factors included parity of the women, the patient's future reproductive expectations, and the reassuring comfort of feminity implicit in the preservation of the monthly menstrual experience.

This is a descriptive study, analyzing the contribution of ruptured uterus to maternal mortality over a ten year period in a rural village in Southeast Nigeria. The study therefore adequately assessed the maternity services available to the population at that period without bias. However the total of 3046 maternities over the period may suggest that a greater number of parturients delivered outside the facility and the cases analyzed might not have been very representative of the population. Again it was not possible to clearly isolate the risk factors associated with maternal death or uterine rupture. The study could not control for confounding 
variables. A case controlled study could have been more appropriate for this. The management modality of cases studied followed the routine protocol of the unit at that time. There was no attempt to randomize the patients into different management protocols to assess the most effective protocol. Such randomisation is often impracticable in such cases of ruptured uterus presenting in very critical clinical conditions.

The high MMR recorded in this review was similar to figures recorded for other rural/semi urban areas of the country ${ }^{3,4,15}$. The $31.9 \%$ contribution to maternal mortality of uterine rupture in our study is comparable to the figure of $28.2 \%$ reported from Sagamu in Southwest Nigeria ${ }^{16}$ but higher than the $7 \quad 10 \%$ observed in other parts of Africa $^{17,18}$

Of the cases reported in 1974 from a rural hospital in Northern Nigeria ${ }^{19}$, the uterus had ruptured in 98 out of 102 patients before arrival. In agreement with the findings of others ${ }^{7.9}$ our cases of uterine rupture occurred mainly in unbooked women. All the studies cited above were descriptive in nature and, as such, shared the corresponding strengths and weaknesses of our own review.

The appallingly high maternal mortality rates associated with uterine rupture in the rural areas may be accounted for mainly by the socioeconomic circumstances, and partly by the cultural characteristics of the people. Contributory factors include poverty, illiteracy, absent or grossly inadequate (and often unaffordable) maternal care and public health services, non existent communication and poor road transportation facilities. The majority of the women (over 39\%) lived outside a $10 \mathrm{~km}$ radius of the hospital (Table II). The impact of road transportation difficulties and absent communication facilities climaxed at night and during the rainy season when many roads became unmotorable. On arrival in the hospital, the patient is frequently unable to meet the financial demands of admission. The grave obstetric emergency is further complicated by the strong cultural aversion of the people to the donation and transfusion of blood. The introduction of free maternal care services has been shown to result in a reduction of maternal mortality ${ }^{3}$. Mortality from

\section{References}

1. Harrison KA. Maternal Mortality in Nigeria. The Real Issues. Afr JReprod Health, 1997; 1(1): 7-13.

2. UNDP. Nigerian Human Development Report 2000/2001. Millenniumedition, United Nations Development Programme, Lagos, Nigeria, 2001.

3. Egwuatu VE. Reflections on Maternal Mortality in Nigeria: The fifth Okoronkwo Kesandu Ogan Memorial Oration. Trop JObstet Gynaecol, 2003;20(1):76-82. ruptured uterus is determined mainly by promptness of resuscitative and definitive management, availability of blood for transfusion, competent surgical intervention and adequate anaesthesia ${ }^{10}$. In this study, all the patients who were operated upon within 6 hours of arrival into the hospital survived, and thereafter mortality rose as the admission - surgical interval increased (Table IV). Lack of blood transfusion services was a major disadvantage in the rural community reviewed. Of the 18 patients who were not transfused with blood, 11 died before surgical intervention was feasible.

Reduction in maternal mortality in the rural areas of Nigeria may be achieved through poverty alleviation, illiteracy eradication, improvement in maternal care, communication, and road transportation facilities, and the establishment of effective blood transfusion services.

The poor utilization of existing meagre maternity and public health services as well as the strong cultural aversion of the people to blood donation and transfusion in the rural areas of Nigeria are of great concern. Research should be intensified in these directions in order to formulate suitable intervention strategy to improve the health seeking behaviour of the rural population.

\section{Conclusion}

In spite of numerous programmes aimed at reducing maternal deaths in sub Saharan Africa, the picture is yet to change. The rural areas are worse affected. Uterine rupture makes large contributions to these appalling figures in these communities. Variables leading to maternal deaths in these areas range from the parturient poor health seeking behaviour, lack of sustainable maternity services, poor capacity to react to obstetric emergencies in the existing facilities including inadequate blood banking services to poor state of infrastructural development, transportation and communication as well as poverty and illiteracy prevalent in the rural communities. Any meaningful programme therefore must be multidisciplinary and make provisions for the above. Meanwhile education and literacy campaigns in the rural areas coupled with economic empowerment will go a long way in that direction.

4. Adamu YM, Salihu HM. Barriers to the use of antenatal and obstetric care services in rural Kano, Nigeria. $J$ Obstet Gynaecol, 2002; 22(6): 600-603.

5. World Health Organization. Reduction of Maternal Mortality A joint WHO/UNFPA/UNICEF/World Bank Statement. World Health Organization, Geneva, 1999;4-19.

6. Hamoud $\mathrm{H}$ and Symonds IM. Acute Abdominal Pain in Pregnancy. ln: Arulkumaran S, Symonds IM and Fowlie A 
(eds), Oxford Handbook of Obstetrics and Gynaecology, New Delhi, India: Oxford University Press, 2004;236.

7. Ogunnowo T, Olayemi $\mathrm{O}$ and Aimakhu CO. Uterine Rupture: University College Hospital Ibadan Experience. West Afr $J$ Med,2003;22(3):236-9.

8. Ogunniyi SO, Makinde $\mathrm{OO}$, Faleyemu BL. Rupture of the gravid uterus in Ile-I fe, Nigeria. Trop Doctor, 1990; 20: 188 9.

9. Ekele BA, Audu LR, Muyibi S. Uterine rupture in Sokoto, Northern Nigeria: Are we winning? Afr J Med Sc, 2000; 29: 191-3.

10. Ande ABA, Okpere EE. Uterine Rupture. In Okpere EE (ed) Clinical Obstetrics. Benin City: Uniben Press, 2003;295-9.

11. Egwuatu VE. Rupture of the gravid uterus: an extra-abdominal presentation. Nig Med J, 1980; 10(5\&6): 234-246.

12. Lister UM. Obstructed labour, a series of 320 cases occurring in 4 years in a hospital in Southern Nigeria. J Obstet Gynaecol BrEmp, 1960;67:188-198.

13. Ola RC, Olamijulo JA. Rupture of the uterus at the Lagos University Teaching Hospital, Lagos, Nigeria. West Afr JMed, 1998; 17(3): 188-193.
14. Johanson R. Ruptured Uterus. In: Edmonds DK (ed) Dewhurst's Textbook of Obsterics and Gynaecology for Postgraduates, $6^{\text {th }}$ edition. London: Blackwell Science Itd, 1999;323-324.

15. Uzoigwe SA, John CT. Maternal Mortality in the University of Port Harcourt Teaching Hospital, Port Harcourt in the last year before the New Millennium. Nig J Med, 2004; 13(1): 32 -35 .

16 Sule-Odu AO. Maternal deaths in Sagamu, Nigeria. Int J Gynecol Obstet, 2000; 69(1): 47-9.

17 Garenne M, Mbaye K, Bah MD, Corra P. Risk factors for maternal mortality: a Case control study in Dakar hospitals (Senegal).Afr J Reprod Health, 1997; 1 (1): 14-24.

18. Phillips JA. Ruptured uterus in Mulangi CCAP Hospital 19741982. Trop Doctor, 1990; 20(4): 175-6.

19. Groen GP. Uterine rupture in rural Nigeria: A review of 144 cases. Obstset Gynaecol, 1974; 44:682-687. 